\title{
Anesthetic postconditioning plus hypothermia following cardiopulmonary resuscitation protects the myocardial ultrastructure by modulating inflammatory events in rabbits
}

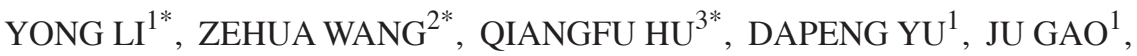 \\ LIUQING YANG ${ }^{1}$, YALI GE ${ }^{1}$, PING CHEN $^{4}$ and LIANG ZONG ${ }^{4-6}$
}

\begin{abstract}
${ }^{1}$ Department of Anesthesiology, Su Bei People's Hospital of Jiangsu Province, Yangzhou University, Yangzhou, Jiangsu 225001; ${ }^{2}$ Department of Anesthesiology, Heji Hospital, Changzhi University, Changzhi Medical College, Changzhi, Shanxi 046000; ${ }^{3}$ Department of Anesthesiology, The Fifth Affiliated Hospital of Zhengzhou University, Zhengzhou, Henan 450052; ${ }^{4}$ Department of Surgery, Su Bei People's Hospital of Jiangsu Province, Yangzhou University, Yangzhou, Jiangsu 225001; ${ }^{5}$ Department of Medical Research Centre, Su Bei People's Hospital of Jiangsu Province, Yangzhou University, Yangzhou, Jiangsu 225001, P.R. China; ${ }^{6}$ Department of Surgery, Graduate School of Medicine, University of Tokyo, Tokyo 113-8655, Japan
\end{abstract}

Received March 9, 2017; Accepted August 30, 2017

DOI: $10.3892 /$ br.2017.976

\begin{abstract}
Myocardial ischemia/reperfusion (I/R) injury is usually caused by resuscitation following cardiac arrest. The aim of the present study was to investigate the influence of sevoflurane postconditioning on the myocardial ultrastructure induced by cardiac arrest and successful resuscitation in mature rabbits. A total of 32 rabbits were randomly and equally divided into an $\mathrm{I} / \mathrm{R}$ group (I/R group), a hypothermia group ( $\mathrm{H}$ group), a sevoflurane postconditioning group (S group), and hypothermia plus sevoflurane postconditioning group ( $\mathrm{H}+\mathrm{S}$ group $)$. Interleukin (IL)-8 and IL-10 levels in blood were evaluated at four different time points ( $1 \mathrm{~h}$ pre-ischemia: T1; 1,2 and $3 \mathrm{~h}$ after reperfusion: T2, T3 and T4, respectively). The myocardial ultrastructure was evaluated by microscope after the rabbits were scarified. Plasma levels of IL-8 and IL-10 increased in all of the groups from T2. However, compared with the I/R group from T3 and T4, downregulation of IL-8 was significant in the $\mathrm{S}$ and $\mathrm{H}+\mathrm{S}$ groups $(\mathrm{P}<0.05)$, and the result of intra-group comparison demonstrated that the level of serum IL-8 was the lowest in the $\mathrm{H}+\mathrm{S}$ group $(\mathrm{P}<0.05)$.
\end{abstract}

Correspondence to: Dr Ju Gao, Department of Anesthesiology, Su Bei People's Hospital of Jiangsu Province, Yangzhou University, 98 NanTong West Road, Yangzhou, Jiangsu 225001, P.R. China E-mail: doctor2227@163.com

Dr Liang Zong, Department of Surgery, Su Bei People's Hospital of Jiangsu Province, Yangzhou University, 98 NanTong West Road, Yangzhou, Jiangsu 225001, P.R. China

E-mail: 250537471@qq.com

*Contributed equally

Key words: cardiopulmonary resuscitation, hypothermia, sevoflurane
By contrast, upregulation of IL-10 was significantly higher in the $\mathrm{S}$ and $\mathrm{H}+\mathrm{S}$ groups $(\mathrm{P}<0.05)$, particularly in the $\mathrm{H}+\mathrm{S}$ group. Notably, ultrastructure damage of the myocardium was significantly lighter, and the structural integrity of the myocardium in the $\mathrm{H}+\mathrm{S}$ group was better when compared with that of the $\mathrm{S}$ group. Thus, sevoflurane postconditioning plus hypothermia protected the myocardial ultrastructure following cardiopulmonary resuscitation by suppressing inflammatory effects.

\section{Introduction}

Myocardial dysfunction after successful cardiopulmonary resuscitation, caused by ischemia/reperfusion (I/R)-injury, is a particularly critical issue in clinical practice $(1,2)$. However, the reported incidence of post-resuscitation myocardial dysfunction is approximately $45-60 \%$ for successfully resuscitated patients (3). Due to a lack of conclusive evidence from experimental animal models, it is still too soon to apply ischemic postconditioning in clinical use.

Previously, certain studies using animal models demonstrated that anesthetic postconditioning is useful for reducing myocardial dysfunction, although the underlying mechanism remains unclear. It was reported that the inflammatory response following brain ischemia was the predominant mechanism causing brain damage (4). Furthermore, numerous studies mentioned that various inflammatory cytokines were released to mediate $\mathrm{I} / \mathrm{R}$ injury and that hypothermia may be a useful method for antagonizing the injury $(1,5,6)$. Notably, hypothermia combined with anesthetic postconditioning demonstrated an improved efficacy for reducing brain I/R injury when compared with hypothermia or anesthetic postconditioning alone.

However, it remains unclear whether cardiac arrest triggers an inflammatory response via a release of inflammatory 
molecules, and whether anesthetic postconditioning combined with hypothermia reduces the cardiac I/R injury by altering the inflammatory response. The present study hypothesized that a combination of anesthetic postconditioning and hypothermia reduces myocardial injury by altering the process of the inflammatory response.

\section{Materials and methods}

Experimental protocol. The present study was approved by the animal investigation committee of Yangzhou University (Yangzhou, China). This experimental study was performed on 32 male and female healthy rabbits, aged four to five months, weighing $2.8-3.2 \mathrm{~kg}$. All the rabbits were fed in individually ventilated cages under a temperature of $25^{\circ} \mathrm{C}$ and a humidity of $75 \%$ (light:dark $=1: 1$ ). The rabbits were randomly and equally divided into an I/R group (I/R group), a hypothermia group (H group), a sevoflurane postconditioning group (S group), and a hypothermia plus sevoflurane postconditioning group $(\mathrm{H}+\mathrm{S}$ group). Cardiac arrest following acute coronary artery ischemia was electrically induced in all of the rabbits. After a $7 \mathrm{~min}$ non-intervention interval, leading to global ischemia, cardiopulmonary resuscitation (CPR) was initiated. Sevoflurane (Jiangsu Hengrui Medicine Co., Ltd., Lianyungang, China) postconditioning was administered at a concentration of $2.5 \%$ for $30 \mathrm{~min}$. Hypothermia was induced by applying a bag containing ice and water on the heart to achieve a temperature of $33^{\circ} \mathrm{C}$. Interleukin (IL)- 8 and IL-10 levels in blood were evaluated at four different time point $(1 \mathrm{~h}$ pre-ischemia: T1; 1, 2 and $3 \mathrm{~h}$ after reperfusion: T2, T3 and $\mathrm{T} 4$, respectively).

Enzyme linked immunosorbent assays (ELISAs) for IL-8 and IL-10. IL-8 and IL-10 levels were determined using ELISA kits from Tecan Group, Ltd., Mannedorf, Switzerland (IL-8: cat. no. M1000; IL-10: cat. no. BE45311) according to the manufacturer's instructions.

Transmission electron microscopy (TEM). The myocardial ultrastructure of rabbits was determined by a TEM (Hitachi, Ltd., Tokyo, Japan). Rabbit hearts were immediately removed following reperfusion and dissected into small pieces (size, $1 \mathrm{~mm}^{3}$ ). Tissues were fixed in $2.5 \%$ glutaraldehyde in $0.1 \mathrm{~mol} / 1$ sodium cacodylate buffer $(\mathrm{pH} 7.4)$ for $4 \mathrm{~h}$, and were consequently post-fixed in $1 \%$ osmium tetroxide in $1 \% \mathrm{~K}_{4}\left[\mathrm{Fe}(\mathrm{CN})_{6}\right]$. Subsequently, several steps were followed, including dehydrating through graded concentrations of ethanol and propylene oxide, embedding in Epon 812 for $24 \mathrm{~h}$ and sectioning using an ultramicrotome. Longitudinal sections $(6 \mu \mathrm{m})$ were placed onto copper grids and stained with uranylacetate and lead nitrate. Each sample was examined under three visual fields (magnification, x8,000).

Statistical analysis. Correlation was analyzed using the Pearson's product-moment correlation coefficients and the P-value was obtained by the Student's t-test. A difference in the mean plasma level of IL-8 and IL-10 was analyzed using the Student's t-test. $\mathrm{P}<0.05$ (two-tailed) was considered to indicate a statistically significant difference (SPSS, Inc., Chicago, IL, USA).

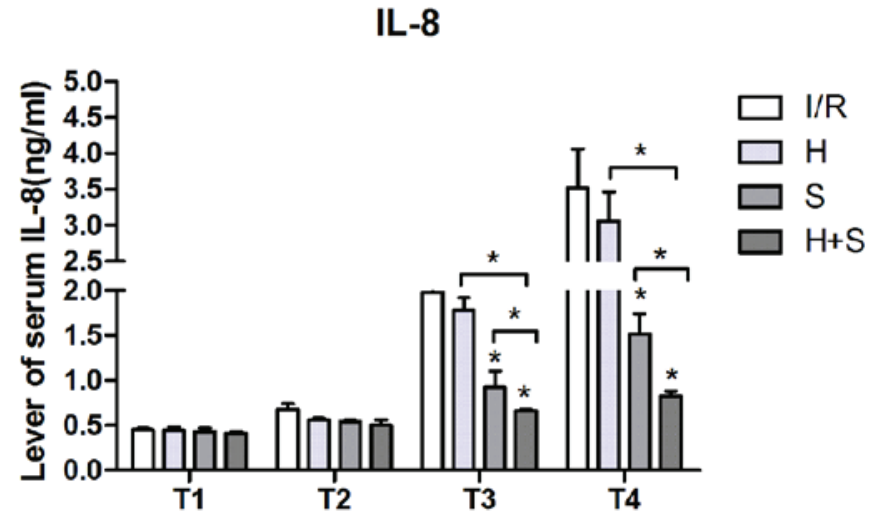

Figure 1. Plasma level of IL-8 in the I/R, H, S and $\mathrm{H}+\mathrm{S}$ groups. IL, interleukin; I/R, ischemia/reperfusion; $\mathrm{H}$, hypothermia; $\mathrm{S}$, sevoflurane.

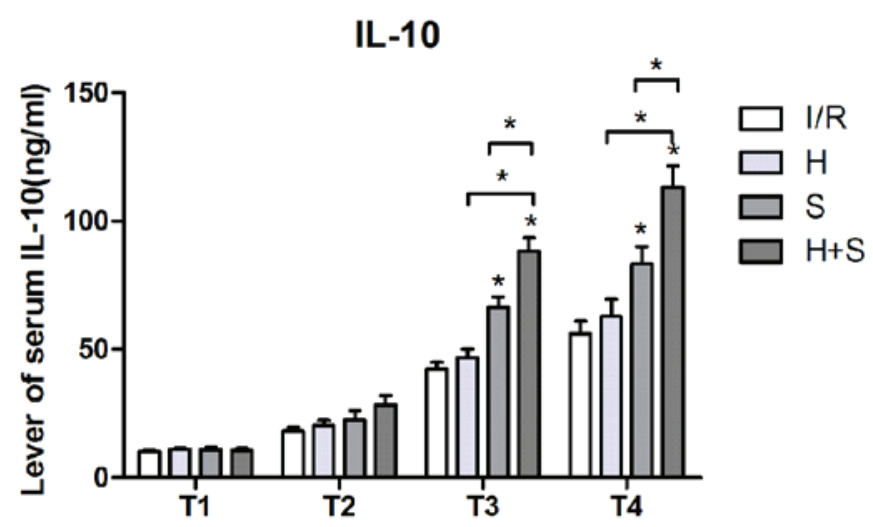

Figure 2. Plasma level of IL-10 in I/R, H, S, and $\mathrm{H}+\mathrm{S}$ groups. IL, interleukin; $\mathrm{I} / \mathrm{R}$, ischemia/reperfusion; $\mathrm{H}$, hypothermia; $\mathrm{S}$, sevoflurane.

\section{Results}

IL- 8 and IL-10 levels. Plasma levels of IL-8 and IL-10 increased in all of the groups from T2. However, compared with the I/R group from T3 and T4, downregulation of IL-8 was significant in the $\mathrm{S}$ and $\mathrm{H}+\mathrm{S}$ groups $(\mathrm{P}<0.05)$, and the result of intra-group comparison demonstrated that the level of serum IL-8 was the lowest in the $\mathrm{H}+\mathrm{S}$ group $(\mathrm{P}<0.05)$. By contrast, upregulation of IL-10 was significantly higher in the $\mathrm{S}$ and $\mathrm{H}+\mathrm{S}$ groups $(\mathrm{P}<0.05)$, particularly in the $\mathrm{H}+\mathrm{S}$ group (Figs. 1 and 2).

Ultrastructure in the cardiac specimens. Using TEM, the I/R group exhibited marked morphological defects and oncosis, which were less pronounced in the $\mathrm{H}, \mathrm{S}$ and $\mathrm{H}+\mathrm{S}$ groups. The $\mathrm{S}$ group demonstrated that sevoflurane postconditioning was effective in preventing ultrastructure damage of cells when compared with the $\mathrm{H}$ group. Notably, the combination of $\mathrm{H}$ and $\mathrm{S}$ demonstrated a synergic effect (Fig. 3).

Association between IL-8 and IL-10, and the myocardial ultrastructure. The increase of IL-10 was negatively correlated with the level of IL-8, indicating that IL-10 may be a key factor for regulating the inflammatory response. Consistently, the group with a higher level of IL-10 exhibited an improved effect when protecting the cell ultrastructure, particularly in the $\mathrm{H}+\mathrm{S}$ group. 

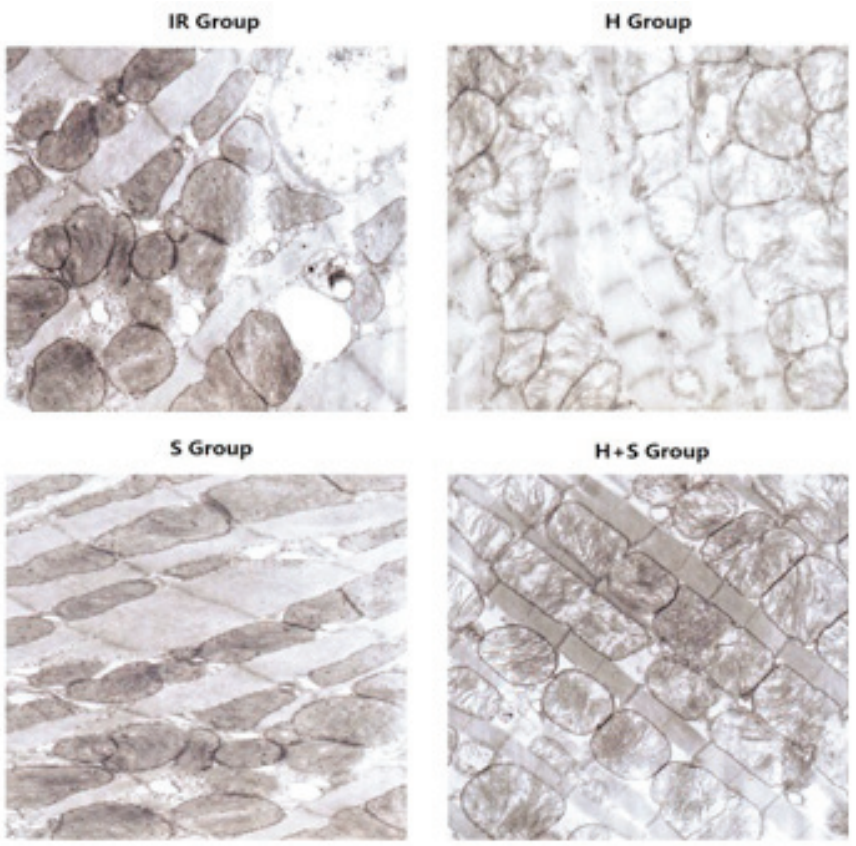

Figure 3. Myocardial ultrastructure under transmission electron microscopy in the $\mathrm{I} / \mathrm{R}, \mathrm{H}, \mathrm{S}$ and $\mathrm{H}+\mathrm{S}$ groups (magnification, $\mathrm{x} 8,000$ ). $\mathrm{R}$, ischemia/reperfusion; H, hypothermia; S, sevoflurane.

\section{Discussion}

In the present study, sevoflurane postconditioning plus hypothermia was identified to be an effective strategy for preventing cardiovascular damage. For the potential mechanism, plasma levels of IL-8 and IL-10 increased in the I/R, H, S, and $\mathrm{S}+\mathrm{H}$ groups from T2. However, compared with the I/R group, IL-8 was not upregulated to the same extent in the $\mathrm{H}, \mathrm{S}$ and $\mathrm{H}+\mathrm{S}$ groups. By contrast, upregulation of IL-10 was stronger in these three groups. Notably, the ultrastructure damage was identified to be significantly less in the $\mathrm{H}+\mathrm{S}$ group, indicating that sevoflurane postconditioning plus hypothermia protected the myocardial ultrastructure following cardiopulmonary resuscitation via suppressing the inflammatory effect.

Myocardial dysfunction and circulatory failure caused by cardiac arrest are the primary reasons contributing to morbidity and mortality following an initially successful cardiopulmonary resuscitation (7). Meybohm et al (6) reported that mild hypothermia contributed to decreased myocardial damage and dysfunction following CPR most probably because hypothermia attenuates immune cell infiltration, apoptosis and pro-inflammatory cytokine expression. Furthermore, it was reported that postconditioning with sevoflurane may protect the myocardium from damage and dysfunction (8). In the current study, a combination of sevoflurane postconditioning and hypothermia was observed to protect the myocardial ultrastructure by modifying the inflammatory response.

Regarding the systemic inflammatory response to cardiac arrest, various different cytokines, including IL-1b, IL-6 and IL-10, have recently been presented in the study of hypothermia to improve dysfunction following cerebral or cardiac arrest (4). However, conflicting results were achieved. Matsui et al (9) and Russwurm et al (10) demonstrated that mild hypothermia contributes to inhibition of IL-10 production in peripheral blood mononuclear cells. However, Callaway et al (11) concluded that hypothermia following cardiac arrest did not alter serum inflammatory cytokines. These data indicate that the role of circulating cytokines may not be specific regarding the protective effect of hypothermia. The current data demonstrated that the combination of mild hypothermia and sevoflurane induced a relatively higher expression of IL-10 following successful resuscitation from cardiac arrest. Furthermore, the expression of IL- 8 was negatively correlated with IL-10, indicating that IL-10 inhibits the inflammatory process. Notably, the myocardial ultrastructure exhibited reduced damage in the $\mathrm{S}$ group.

In conclusion, the findings clearly demonstrate that sevoflurane postconditioning combined with hypothermia confers a protective effect to the rabbit myocardial ultrastructure against myocardial I/R injury. Inflammatory cytokines were involved in the underlying mechanism of sevoflurane postconditioning plus hypothermia against myocardial I/R injury by inhibiting inflammatory effects. Therefore, these data provide mechanistic insights into the cardioprotection of sevoflurane postconditioning plus hypothermia, and provides a potential therapeutic strategy to treat perioperative ischemic events. However, further clinical work is needed for the application in patients following cardiopulmonary resuscitation.

\section{References}

1. Fagundes CT, Amaral FA, Souza AL, Vieira AT, Xu D, Liew FY, Souza DG and Teixeira MM: ST2, an IL-1R family member, attenuates inflammation and lethality after intestinal ischemia and reperfusion. J Leukoc Biol 81: 492-499, 2007.

2. Pepine CJ and Nichols WW: The pathophysiology of chronic ischemic heart disease. Clin Cardiol 30 (Suppl 1): I4-I9, 2007.

3. Neumar RW, Nolan JP, Adrie C, Aibiki M, Berg RA, Böttiger BW, Callaway C, Clark RS, Geocadin RG, Jauch EC, et al: Post-cardiac arrest syndrome: Epidemiology, pathophysiology, treatment, and prognostication. A consensus statement from the International Liaison Committee on Resuscitation (American Heart Association, Australian and New Zealand Council on Resuscitation, European Resuscitation Council, Heart and Stroke Foundation of Canada, InterAmerican Heart Foundation, Resuscitation Council of Asia, and the Resuscitation Council of Southern Africa); the American Heart Association Emergency Cardiovascular Care Committee; the Council on Cardiovascular Surgery and Anesthesia; the Council on Cardiopulmonary, Perioperative, and Critical Care; the Council on Clinical Cardiology; and the Stroke Council. Circulation 118: 2452-2483, 2008.

4. Meybohm P, Gruenewald M, Zacharowski KD, Albrecht M, Lucius R, Fösel N, Hensler J, Zitta K and Bein B: Mild hypothermia alone or in combination with anesthetic postconditioning reduces expression of inflammatory cytokines in the cerebral cortex of pigs after cardiopulmonary resuscitation. Crit Care 14: R21, 2010.

5. Grootjans J, Lenaerts K, Derikx JP, Matthijsen RA, de Bruïne AP, van Bijnen AA, van Dam RM, Dejong CH and Buurman WA: Human intestinal ischemia-reperfusion-induced inflammation characterized: Experiences from a new translational model. Am J Pathol 176: 2283-2291, 2010.

6. Meybohm P, Gruenewald M, Albrecht M, Zacharowski KD, Lucius R, Zitta K, Koch A, Tran N, Scholz J and Bein B: Hypothermia and postconditioning after cardiopulmonary resuscitation reduce cardiac dysfunction by modulating inflammation, apoptosis and remodeling. PLoS One 4: e7588, 2009.

7. Oddo M, Ribordy V,Feihl F, Rossetti AO, Schaller MD, Chioléro R and Liaudet L: Early predictors of outcome in comatose survivors of ventricular fibrillation and non-ventricular fibrillation cardiac arrest treated with hypothermia: A prospective study. Crit Care Med 36: 2296-2301, 2008. 
8. Albrecht M, Gruenewald M, Zitta K, Zacharowski K, Scholz J, Bein B and Meybohm P: Hypothermia and anesthetic postconditioning influence the expression and activity of small intestinal proteins possibly involved in ischemia/reperfusion-mediated events following cardiopulmonary resuscitation. Resuscitation 83 : 113-118, 2012.

9. Matsui T, Ishikawa T, Takeuchi H, Tsukahara M and Maekawa T: Mild hypothermia inhibits IL-10 production in peripheral blood mononuclear cells. Acta Anaesthesiol Scand 48: 205-210, 2004.
10. Russwurm S, Stonāns I, Schwerter K, Stonāne E, Meissner W and Reinhart K: Direct influence of mild hypothermia on cytokine expression and release in cultures of human peripheral blood mononuclear cells. J Interferon Cytokine Res 22: 215-221, 2002. 11. Callaway CW, Rittenberger JC, Logue ES and McMichael MJ: Hypothermia after cardiac arrest does not alter serum inflammatory markers. Crit Care Med 36: 2607-2612, 2008. 